\title{
有限域上长为 $2^{m} p^{n}$ 的负循环码
}

\author{
沈如林 ${ }^{(1)}$ (3)，张光辉 ${ }^{(2)}$ ，陈博聪 ${ }^{(3)}$
}

(1) 湖北民族学院数学系, 恩施 445000 ;

(2) 洛阳师范学院数学科学学院, 洛阳 471022 ;

(3) 华中师范大学数学与统计学学院, 武汉 430079

E-mail: rulinshen@gmail.com, zghui2012@126.com, bocong_chen@yahoo.com

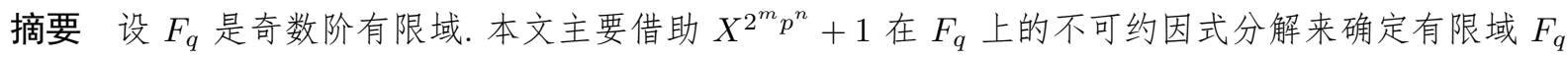
上所有长为 $2^{m} p^{n}$ 的负循环码和自对偶的负循环码的生成多项式,这里 $p$ 是 $q-1$ 的奇素因子, $m$ 和 $n$ 是正整数.

关键词 负循环码 不可约分解 生成多项式 对偶码

MSC (2010) 主题分类 $11 \mathrm{~T} 71,94 \mathrm{~B} 15$

\section{1 引言}

负循环码 ${ }^{[1,2]}$ 的研究始于 19 世纪 60 年代, 随后由于其理论的重要性和应用的广泛性而受到关 注. 人们关注的焦点之一在于确定负循环码和自对偶的负循环码的代数结构 [3-14].

Dinh ${ }^{[9]}$ 给出了特征为奇素数 $p$ 的有限域 $F_{p^{m}}$ 上长为 $2 p^{s}$ 的负循环码的生成多项式, 同时也研究 了长为 $2 p^{s}$ 的自对偶的负循环码. Chen 等人 ${ }^{[5]}$ 得到了有限域 $F_{p^{m}}$ 上长为 $\ell^{t} p^{s}$ 的负循环码的生成多 项式, 这里 $\ell$ 是一个与 $p$ 互异的素数.

设 $F_{q}$ 是一个 $q$ 阶有限域, 这里 $q$ 是奇数; $N$ 是一个与 $q$ 互素的正整数. $F_{q}$ 上任一个长为 $N$ 的负 循环码恰等同于商代数 $F_{q}[X] /\left\langle X^{N}+1\right\rangle$ 的一个理想. 既然 $F_{q}[X] /\left\langle X^{N}+1\right\rangle$ 的每一个理想由 $X^{N}+1$ 的一个首一的因式所生成, 那么, 由 $X^{N}+1$ 在 $F_{q}[X]$ 中的不可约因式分解就完全确定了 $F_{q}$ 上所有长 为 $N$ 的负循环码. 设 $C=\langle g(X)\rangle$ 是 $F_{q}$ 上一个长为 $N$ 的负循环码, 这里 $g(X)$ 是 $X^{N}+1$ 的首一因 式. 令

$$
h(X)=\frac{X^{N}+1}{g(X)}, \quad h^{*}(X)=h(0)^{-1} X^{\operatorname{deg} h} h\left(\frac{1}{X}\right)
$$

是 $h(X)$ 的互反多项式, 则负循环码 $C$ 的对偶码 $C^{\perp}$ 也是 $F_{q}$ 上的一个长为 $N$ 的负循环码, 其生成多 项式为 $h^{*}(X)$. 易知, $C$ 是自对偶的当且仅当 $g(X)=h^{*}(X)$. 由此可知, 为了确定 $F_{q}$ 上长为 $N$ 的负 循环码的生成多项式, 我们需要给出 $X^{N}+1$ 在 $F_{q}$ 上的不可约因式分解, 以及每一个不可约因式的互 反多项式.

显然, $\left(X^{N}+1\right)\left(X^{N}-1\right)=X^{2 N}-1$. 而 $X^{2 N}-1$ 在 $F_{q}$ 上的不可约因式可以由模 $2 N$ 的 $q$ - 分圆

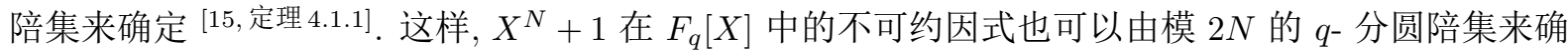


定, 也就是说, 通过模 $2 N$ 的 $q$ - 分圆陪集可以确定 $F_{q}$ 上长为 $N$ 的负循环码的生成多项式. 但是, 一 般情况下, 很难用这种方法来确定自对偶的负循环码的生成多项式. 因为在这种情况下我们不知道在 $X^{N}+1$ 的一个不可约分解中, 任一个不可约因式的互反多项式等于哪一个不可约因式.

但是, 对于特殊的 $N$, 上面提到的方法是可行的. 最近, Bakshi 和 Raka ${ }^{[16]}$ 利用模 $2^{n+1}$ 的分圆陪 集确定了奇特征的有限域 $F$ 上长为 $2^{n}$ 的所有负循环码的生成多项式, 并且也确定了 $F$ 上的自对偶 负循环码的生成多项式及其它们的个数; 文献 [17] 利用同样的方法确定了模 $4 p^{n}$ 的 $q$ - 分圆陪集, 这 里 $p$ 是与 $q$ 互素的奇素数, 由此确定了 $F_{q}$ 上长为 $2 p^{n}$ 的所有负循环码和自对偶的负循环码.

本文主要研究 $F_{q}$ 上长为 $2^{m} p^{n}$ 的负循环码, 这里 $p$ 是 $q-1$ 的奇素因子; $m$ 和 $n$ 是两个正整数. 我们得到了 $F_{q}$ 上长为 $2^{m} p^{n}$ 的负循环码的生成多项式, 以及所有自对偶负循环码的生成多项式和个 数; 不用分圆陪集, 我们给出 $X^{2^{m} p^{n}}+1$ 在 $F_{q}$ 上的不可约分解. 值得注意的是, 若 $4 \mid(q-1)$, 则 $F_{q}$ 上 长为 $2^{m} p^{n}$ 的负循环码的生成多项式的每一个不可约因式都是二项式, 否则, 均是三项式. 另外, 我们 也得到若 $M$ 是一个正整数, 它的每一个素因子均整除 $q-1$, 且 $4 \mid(q-1)$, 则 $X^{M}-\lambda$ 的不可约因子 都是二项式, 这里 $\lambda$ 是 $F_{q}$ 中的任一个非零元素. 而不可约二项式的应用参见文献 [18].

\section{2 预备知识}

本文用 $F_{q}$ 表示一个奇数阶有限域, $F_{q}^{*}$ 表示由 $F_{q}$ 全部非零元素所构成的乘法群. 任取 $\beta \in F_{q}^{*}$, $\operatorname{ord}(\beta)$ 表示 $\beta$ 在乘法群 $F_{q}^{*}$ 中阶. 因此, $\operatorname{ord}(\beta)$ 是 $q-1$ 的一个因子, $\beta$ 称为一个本原 $\operatorname{ord}(\beta)$ 次单位 根. 众所周知, $F_{q}^{*}$ 是一个 $q-1$ 阶循环群, 也就是说, $F_{q}^{*}$ 是由一个本原 $(q-1)$ 次单位根 $\xi$ 所生成, 我 们记作 $F_{q}^{*}=\langle\xi\rangle$. 对任一个整数 $k$, 我们有 $\operatorname{ord}\left(\xi^{k}\right)=\frac{q-1}{\operatorname{gcd}(k, q-1)}$, 这里 $\operatorname{gcd}(k, q-1)$ 表示 $k$ 和 $q-1$ 的 最大公因式.

设 $N$ 是一个与 $q$ 互素的正整数, $F_{q}^{N}$ 是 $F_{q}$ 上全部 $N$ - 元数组所构成的 $F_{q^{-}}$线性空间. 设 $C$ 是 $F_{q}$ 上一个长为 $N$ 的线性码, 如果对每一个 $\left(c_{0}, c_{1}, \ldots, c_{N-1}\right) \in C$, 都有 $\left(-c_{N-1}, c_{0}, \ldots, c_{N-2}\right) \in C$, 那 么, 我们称 $C$ 为一个负循环码. 对 $F_{q}$ 上的一个长为 $N$ 的负循环码 $C$, 我们定义 $C$ 的对偶码为 $C^{\perp}=\left\{u \in F_{q}^{N} \mid u \cdot v=0, \forall v \in C\right\}$, 这里 $u \cdot v$ 表示 $F_{q}^{N}$ 中 $u$ 和 $v$ 的标准 Euclid 内积. 不难证明,负循 环码 $C$ 的对偶码 $C^{\perp}$ 也是一个负循环码. 如果 $C=C^{\perp}$, 则称 $C$ 是自对偶的.

我们已经知道, $F_{q}$ 上长为 $N$ 的负循环码 $C$ 等同于商代数 $F_{q}[X] /\left\langle X^{N}+1\right\rangle$ 的一个理想, 它由 $X^{N}+1$ 的唯一一个首一因式 $g(X)$ 所生成, 此时, $g(X)$ 称为 $C$ 的生成多项式, 我们记作 $C=\langle g(X)\rangle$. 特别地, $X^{N}+1$ 在 $F_{q}[X]$ 中的不可约多项式的因式分解可以完全确定 $F_{q}$ 上长为 $N$ 的全部负循环码. 设 $h(X)=\frac{X^{N}+1}{g(X)}$, 则 $C^{\perp}$ 的生成多项式是 $h^{*}(X)$, 这里 $h^{*}(X)=h(0)^{-1} X^{\operatorname{deg} h} h\left(\frac{1}{X}\right)$, 称为 $h(X)$ 的互反 多项式. 注意 $h^{*}(X)$ 是首一多项式并且整除 $X^{N}+1$. 如果一个多项式等于它的互反多项式, 那么, 我 们就称为自反的.

下面的讨论刻画了 $F_{q}$ 上所有长为 $N$ 的自对偶的负循环码. 记

$$
X^{N}+1=f_{1}(X) f_{2}(X) \cdots f_{u}(X) h_{1}(X) h_{1}^{*}(X) h_{2}(X) h_{2}^{*}(X) \cdots h_{v}(X) h_{v}^{*}(X),
$$

这里 $f_{i}(X)$ 是一个 $F_{q}$ 上的首一的自反的不可约多项式, $h_{j}(X)$ 和它的互反多项式 $h_{j}^{*}(X)$ 都是 $F_{q}$ 上 的首一的不可约多项式.

设 $C$ 是 $F_{q}$ 上一个长为 $N$ 的负循环码, 它的生成生成多项式是 $g(X)$. 令

$$
g(X)=f_{1}(X)^{\tau_{1}} \cdots f_{u}(X)^{\tau_{u}} h_{1}(X)^{\nu_{1}} h_{1}^{*}(X)^{\omega_{1}} \cdots h_{v}(X)^{\nu_{v}} h_{v}^{*}(X)^{\omega_{v}},
$$


这里 $\tau_{i}, \nu_{j}, \omega_{k}$ 等于 0 或 1 , 则

$$
h(X)=f_{1}(X)^{1-\tau_{1}} \cdots f_{u}(X)^{1-\tau_{u}} h_{1}(X)^{1-\nu_{1}} h_{1}^{*}(X)^{1-\omega_{1}} \cdots h_{v}(X)^{1-\nu_{v}} h_{v}^{*}(X)^{1-\omega_{v}} .
$$

因此,

$$
h^{*}(X)=f_{1}(X)^{1-\tau_{1}} \cdots f_{u}(X)^{1-\tau_{u}} h_{1}(X)^{1-\omega_{1}} h_{1}^{*}(X)^{1-\nu_{1}} \cdots h_{v}(X)^{1-\omega_{v}} h_{v}^{*}(X)^{1-\nu_{v}} .
$$

由此得到 $C$ 是 $F_{q}$ 上的一个长为 $N$ 的自对偶的负循环码当且仅当 $u=0$ 和 $\omega_{j}+\nu_{j}=1,1 \leqslant j \leqslant v$, 此 时, $N$ 必是一个偶数, 且

$$
C=\langle g(X)\rangle=\left\langle\prod_{j=1}^{v}\left(h_{j}(X)^{\varepsilon_{j}} h_{j}^{*}(X)\right)^{1-\varepsilon_{j}}\right\rangle,
$$

这里 $\varepsilon_{j}$ 等于 0 或 1 . 特别地, $F_{q}$ 上共有 $2^{v}$ 个长为 $N$ 的自对偶的负循环码.

鉴于上面的讨论, 我们可以通过写出 $X^{2^{m} p^{n}}+1$ 在 $F_{q}$ 上形如 (2.1) 的不可约因式分解, 来确定 $F_{q}$ 上所有长为 $2^{m} p^{n}$ 的自对偶的负循环码.

值得注意的是, 在一些特殊情形下 $X^{N}+1$ 在 $F_{q}[X]$ 中的不可约因式分解可以给出刻画. 为了便 于引用, 我们把它写作注 2.1 .

注 2.1 设 $q \equiv 3(\bmod 4), m$ 是一个正整数. 在这些条件下, $X^{2^{m}}+1$ 在 $F_{q}$ 上的不可约分解已完 全确定 (参见文献 [19, 定理 1] 或 [5, 注 2.2]). 注意到 $4 \mid(q+1)$, 则存在一个整数 $a \geqslant 2$, 使得 $2^{a} \|(q+1)$, 这里符号 $2^{a} \|(q+1)$ 表示 $2^{a} \mid(q+1)$, 但是 $2^{a+1} \nmid(q+1)$. 令 $H_{1}=\{0\}$, 递归定义

$$
H_{i}=\left\{ \pm\left(\frac{h+1}{2}\right)^{\frac{q+1}{4}} \mid h \in H_{i-1}\right\},
$$

这里 $i=2,3, \ldots, a-1$. 再令

$$
H_{a}=\left\{ \pm\left(\frac{h-1}{2}\right)^{\frac{q+1}{4}} \mid h \in H_{a-1}\right\},
$$

则对每一个 $1 \leqslant m \leqslant(a-1),\left|H_{m}\right|=2^{m-1}$, 并且 $X^{2^{m}}+1$ 在 $F_{q}$ 上的不可约分解如下:

$$
X^{2^{m}}+1= \begin{cases}\prod_{h \in H_{m}}\left(X^{2}-2 h X+1\right), & \text { 若 } 1 \leqslant m \leqslant(a-1), \\ \prod_{h \in H_{a}}\left(X^{2^{m-a+1}}-2 h X^{2^{m-a}}-1\right), & \text { 若 } m \geqslant a .\end{cases}
$$

最后回顾有限域 $F_{q}$ 上二项式的一个不可约判定准则 (参见文献 [20, 定理 3.75] 或 [21, 定理 10.7]). 为方便引用, 我们把它作为一个注 2.2 .

注 2.2 设正整数 $k \geqslant 2$. 对任一个 $\gamma \in F_{q}^{*}, \operatorname{ord}(\gamma)=e$, 二项式 $X^{k}-\gamma$ 在 $F_{q}$ 上不可约当且仅当 下面的两个条件均成立:

(1) $k$ 的每一个素因子均整除 $e$, 但不整除 $(q-1) / e$;

(2) 若 $4 \mid k$, 则 $4 \mid(q-1)$.

\section{3 有限域 $F_{q}$ 上长为 $2^{m} p^{n}$ 的负循环码}

设 $F_{q}$ 是一个奇数阶有限域, 它的乘法群 $F_{q}^{*}=\langle\xi\rangle$, 其中 $\xi$ 是一个本原 $q-1$ 次单位根. 本节假设 $p$ 是 $q-1$ 的一个素数因子. 记 $q-1=2^{s} p^{t} c$, 其中 $s$ 和 $t$ 是正整数, 并且 $\operatorname{gcd}(2 p, c)=1$. 
设 $m$ 和 $n$ 是两个正整数. 显然, 有限域 $F_{q}$ 上长为 $2^{m} p^{n}$ 的负循环码的生成多项式完全由 $X^{2^{m}} p^{n}+1$ 在 $F_{q}$ 上的不可约因式所决定. 我们将根据下面两种情形来讨论有限域 $F_{q}$ 上长为 $2^{m} p^{n}$ 的负循环码 的生成多项式的构造, $s \geqslant 2$, 即 $4 \mid(q-1)$ 和 $s=1$, 即 $4 \nmid(q-1)$.

\section{1 有限域 $F_{q}$ 上长为 $2^{m} p^{n}$ 的负循环码, 这里 $4 \mid(q-1)$}

我们假设 $q-1=2^{s} p^{t} c, s \geqslant 2$. 首先有下面的引理.

引理 3.1 符号如上. 假设 $n \leqslant t$, 那么, $X^{2^{m} p^{n}+1}$ 在 $F_{q}$ 上的不可约分解如下:

$$
X^{2^{m} p^{n}}+1= \begin{cases}\prod_{\substack{i=1 \\ \text { 小ं } \\ 2^{s} p^{n}}}^{2^{m+1} p^{n}}\left(X-\sigma^{i}\right), & \text { 如果 } m<s, \\ \prod_{\substack{j=1 \\ 2 \nmid j}}\left(X^{2^{m-s+1}}-\eta^{j}\right), & \text { 如果 } m \geqslant s,\end{cases}
$$

这里 $\sigma=\xi^{2^{s-m-1} p^{t-n} c}$ 是一个本原 $2^{m+1} p^{n}$ 次单位根 $(m<s), \eta=\xi^{p^{t-n} c}$ 是一个本原 $2^{s} p^{n}$ 次单位根 $(m \geqslant s)$.

证明 如果 $m<s$, 容易验证 $X^{2^{m}} p^{n}+1$ 有如下的分解:

$$
X^{2^{m} p^{n}}+1=\prod_{\substack{i=1 \\ 2 \nmid i}}^{2^{m+1} p^{n}}\left(X-\sigma^{i}\right)
$$

这里 $\sigma=\xi^{2^{s-m-1} p^{t-n} c}$ 是一个本原 $2^{m+1} p^{n}$ 次单位根.

对于 $m \geqslant s$ 的情形, 我们取一个 $j$, 使得 $1 \leqslant j \leqslant 2^{s} p^{n}$ 且 $2 \nmid j$. 令 $\eta=\xi^{p^{t-n} c}$, 则 $\eta$ 是一个本原 $2^{s} p^{n}$ 次单位根. 因此, $\operatorname{ord}\left(\eta^{j}\right)=\frac{2^{s} p^{n}}{\operatorname{gcd}\left(j, 2^{s} p^{n}\right)}$ 且 $2^{s} \mid \operatorname{ord}\left(\eta^{j}\right)$. 根据注 2.1 , 我们知道 $X^{2^{m-s+1}}-\eta^{j}$ 是 $F_{q}[X]$ 中的 不可约多项式. 假设 $\beta$ 是 $X^{2^{m-s+1}}-\eta^{j}$ 的任一个根, 则 $\beta^{2^{m-s+1}}=\eta^{j}$. 由 $\eta=\xi^{p^{t-n} c}$ 是一个本原 $2^{s} p^{n}$ 次单位根, 因此, $\beta^{2^{m} p^{n}}=\eta^{j 2^{s-1} p^{n}}=-1$. 由此可得 $X^{2^{m-s+1}}-\eta^{j}$ 是 $X^{2^{m} p^{n}}+1$ 的一个首一的不可约 因式. 因此,

$$
f(X)=\prod_{\substack{j=1 \\ 2 \nmid j}}^{2^{s} p^{n}}\left(X^{2^{m-s+1}}-\eta^{j}\right)
$$

是 $X^{2^{m} p^{n}}+1$ 的一个首一因式. 再注意到 $f(X)$ 的次数等于 $2^{m-s+1} \cdot 2^{s-1} p^{n}=2^{m} p^{n}$, 即得引理的第二 部分.

引理 3.2 符号如上.

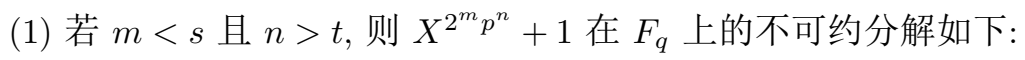

$$
X^{2^{m} p^{n}}+1=\prod_{\substack{k=1 \\ 2 \nmid k}}^{2^{m+1} p^{t}}\left(X-\sigma_{1}^{k}\right) \cdot \prod_{i=0}^{n-t-1} \prod_{\substack{j=1 \\ p \nmid j, 2 \nmid j}}^{2^{m+1} p^{t}}\left(X^{p^{n-t-i}}-\sigma_{1}^{j}\right),
$$

这里 $\sigma_{1}=\xi^{2^{s-m-1} c}$ 是一个本原 $2^{m+1} p^{t}$ 次单位根;

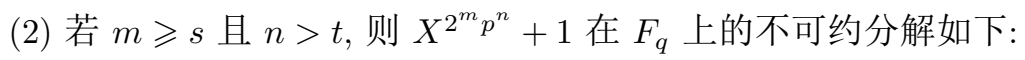

$$
X^{2^{m} p^{n}}+1=\prod_{\substack{u=1 \\ 2 \nmid u}}^{2^{s} p^{t}}\left(X^{2^{m+1-s}}-\theta^{u}\right) \cdot \prod_{j=1}^{n-t} \prod_{\substack{r=1 \\ 2 \nmid r, p \nmid r}}^{2^{s} p^{t}}\left(X^{2^{m+1-s} p^{j}}-\theta^{r}\right),
$$


这里 $\theta=\xi^{c}$ 是一个本原 $2^{s} p^{t}$ 次单位根.

证明 (1) 显然, $\sigma_{1}=\xi^{2^{s-m-1} c}$ 是一个本原 $2^{m+1} p^{t}$ 次单位根. 根据注 2.2 , 我们知道 (3.1) 的右边

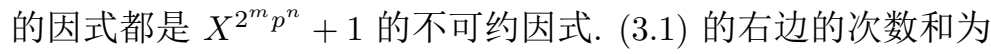

$$
2^{m} p^{t}+\left(p^{n-t}+p^{n-t-1}+\cdots+p\right)\left(2^{m+1} p^{t}-2^{m} p^{t}-2^{m+1} p^{t-1}+2^{m} p^{t-1}\right)=2^{m} p^{n} .
$$

这样就得到 (1). 同理可证 (2).

下面的定理刻画了有限域 $F_{q}$ 上所有长为 $2^{m} p^{n}$ 的负循环码, 这里 $p$ 是 $q-1$ 的一个奇素因子, 且 $4 \mid(q-1)$. 为方便记, 我们总假设下面的 $\varepsilon_{i}^{j}$ 等于 0 或 $1, i$ 和 $j$ 分别取遍它们的下标和上标.

定理 3.3 设 $F_{q}$ 是 $q$ 元有限域且 $4 \mid(q-1)$, 则 $F_{q}$ 上全部的长为 $2^{m} p^{n}$ 的负循环码为

(1) 若 $m<s$ 且 $n \leqslant t$, 则

$$
\left\langle\prod_{\substack{i=1 \\ 2 \nmid i}}^{2^{m+1} p^{n}}\left(X-\sigma^{i}\right)^{\varepsilon_{i}}\right\rangle
$$

这里 $\sigma=\xi^{2^{s-m-1}} p^{t-n} c$ 是一个本原 $2^{m+1} p^{n}$ 次单位根;

(2) 若 $m \geqslant s$ 且 $n \leqslant t$, 则

$$
\left\langle\prod_{\substack{j=1 \\ 2 \nmid j}}^{2^{s} p^{n}}\left(X^{2^{m-s+1}}-\eta^{j}\right)^{\varepsilon_{j}}\right\rangle
$$

这里 $\eta=\xi^{p^{t-n} c}$ 是一个本原 $2^{s} p^{n}$ 次单位根;

(3) 若 $m<s$ 且 $n>t$, 则

$$
\left\langle\prod_{\substack{k=1 \\ 2 \nmid k}}^{2^{m+1} p^{t}}\left(X-\sigma_{1}^{k}\right)^{\varepsilon_{k}} \cdot \prod_{i=0}^{n-t-1} \prod_{\substack{j=1 \\ p \nmid j, 2 \nmid j}}^{2^{m+1} p^{t}}\left(X^{p^{n-t-i}}-\sigma_{1}^{j}\right)^{\varepsilon_{i}^{j}}\right\rangle,
$$

这里 $\sigma_{1}=\xi^{2^{s-m-1} c}$ 是一个本原 $2^{m+1} p^{t}$ 次单位根;

(4) 若 $m \geqslant s$ 且 $n>t$, 则

$$
\left\langle\prod_{\substack{u=1 \\ 2 \nmid u}}^{2^{s} p^{t}}\left(X^{2^{m+1-s}}-\theta^{u}\right)^{\varepsilon_{u}} \cdot \prod_{j=1}^{n-t} \prod_{\substack{r=1 \\ 2 \nmid r, p \nmid r}}^{2^{s} p^{t}}\left(X^{2^{m+1-s}} p^{j}-\theta^{r}\right)^{\varepsilon_{j}^{r}}\right\rangle,
$$

这里 $\theta=\xi^{c}$ 是一个本原 $2^{s} p^{t}$ 次单位根.

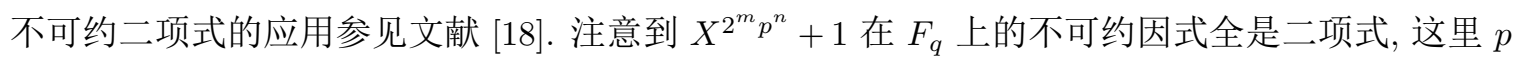
是 $q-1$ 的奇素数因子并且 $4 \mid(q-1)$. 一般地, 我们有下面的结果.

命题 3.4 设 $M>1$ 是一个正整数, 并且满足 $M$ 的每一个素因子均整除 $q-1$. 若 $4 \mid(q-1)$, 则 对每一个 $\lambda \in F_{q}^{*}, X^{M}-\lambda$ 的不可约因式全是二项式.

证明 我们对 $M$ 的素因子个数进行归纳 (计算重数). 如果 $M$ 是一个素数, 根据文献 [5, 推论 4.3] 即得所需结论. 任取 $\lambda \in F_{q}^{*}$. 如果 $X^{M}-\lambda$ 是 $F_{q}[X]$ 中的一个不可约多项式, 那么, 结论也成立. 因此, 我们可以假设 $X^{M}-\lambda$ 在 $F_{q}[X]$ 中是可约的. 根据注 2.2, 我们知道注 2.2 中的条件 (1) 不成立. 这样 就存在 $M$ 的一个素因子 $r_{0}$, 满足 $r_{0} \mid \frac{q-1}{\operatorname{ord}(\lambda)}$ 或者 $r_{0} \nmid \operatorname{ord}(\lambda)$. 令 $\operatorname{ord}(\lambda)=n^{\prime}$ 且 $M=r_{0} m^{\prime}$. 若 $r_{0} \mid \frac{q-1}{n^{\prime}}$, 则 $n^{\prime} \mid \frac{q-1}{r_{0}}$. 因为 $F_{q}^{*}$ 是一个阶为 $q-1$ 的循环群, 所以, $F_{q}^{*}$ 有唯一的阶为 $\frac{q-1}{r_{0}}$ 的子群, 即为 $\left\langle\xi^{r_{0}}\right\rangle$. 又 
$n^{\prime} \mid \frac{q-1}{r_{0}}$ 和 $\operatorname{ord}(\lambda)=n^{\prime}$, 故 $\lambda \in\left\langle\xi^{r_{0}}\right\rangle$. 因此, 存在某个元素 $\mu \in F_{q}^{*}$, 使得 $\lambda=\mu^{r_{0}}$. 既然 $r_{0}$ 是 $q-1$ 的一 个素因子, 我们可以在 $F_{q}$ 中取一个本原 $r_{0}$ 次单位根 $\alpha$. 这样,

$$
X^{M}-\lambda=X^{r_{0} m^{\prime}}-\lambda=X^{r_{0} m^{\prime}}-\mu^{r_{0}}=\prod_{i=0}^{r_{0}-1}\left(X^{m^{\prime}}-\mu \alpha^{i}\right) .
$$

对 $m^{\prime}$ 用归纳假设, 即得对每一个 $0 \leqslant i \leqslant r_{0}-1, X^{m^{\prime}}-\mu \alpha^{i}$ 的不可约因式全是二项式, 即得在这种情形 下结论成立. 现在再假设 $r_{0} \nmid n^{\prime}$, 则存在两个整数 $a$ 和 $b$, 使得 $a n^{\prime}+b r_{0}=1$. 因此, $\lambda=\lambda^{a n^{\prime}+b r_{0}}=\lambda^{b r_{0}}$. 令 $\lambda^{b}=\gamma$, 我们就有 $\lambda=\gamma^{r_{0}}$. 类似于对前一种情形的讨论, 即得所想要的结果.

\section{2 有限域 $F_{q}$ 上长为 $2^{m} p^{n}$ 的负循环码, 这里 $4 \nmid(q-1)$}

设 $F_{q}$ 是一个奇数阶有限域, 如前 $F_{q}^{*}=\langle\xi\rangle$. 本节将给出 $F_{q}$ 上全部的长为 $2^{m} p^{n}$ 的负循环码的生 成多项式, 这里 $p$ 是 $q-1$ 的素因子, 且 $4 \nmid(q-1)$. 进一步我们假设 $2^{a} \|(q+1)$, 其中 $2^{a} \|(q+1)$ 表示 $2^{a} \mid(q+1)$, 但是 $2^{a+1} \nmid(q+1)$. 显然, $a \geqslant 2$. 回忆 $q-1=2 p^{t} c$, 这里 $t$ 是一个正整数且 $\operatorname{gcd}(2 p, c)=1$.

我们先给出 $X^{p^{n}}+1$ 在 $F_{q}$ 上的不可约分解.

引理 3.5 设 $p$ 是 $q-1$ 的一个素因子, $\delta=\xi^{2 c}$ 是一个本原 $p^{t}$ 次单位根, 则 $X^{p^{n}}+1$ 在 $F_{q}$ 上的 不可约分解如下:

$$
X^{p^{n}}+1= \begin{cases}\prod_{j=0}^{p^{n}-1}\left(X+\vartheta^{j}\right), & \text { 若 } n \leqslant t, \\ \prod^{t}-1 & \\ \prod_{k=0}\left(X+\delta^{k}\right) \cdot \prod_{j=1}^{n-t} \prod_{\substack{i=1 \\ p \nmid i}}^{p^{t}}\left(X^{p^{j}}+\delta^{i}\right), & \text { 若 } n>t,\end{cases}
$$

这里 $\vartheta=\xi^{2 p^{t-n} c}$ 是一个本原 $p^{n}$ 次单位根 $(n \leqslant t)$.

证明 类似于引理 3.1 的证明.

根据引理 3.5 , 我们容易得到因式分解

$$
X^{2^{m} p^{n}}+1= \begin{cases}\prod_{j=0}^{p^{n}-1}\left(X^{2^{m}}+\vartheta^{j}\right), & \text { 若 } n \leqslant t, \\ p^{t}-1 & \\ \prod_{k=0}\left(X^{2^{m}}+\delta^{k}\right) \cdot \prod_{j=1}^{n-t} \prod_{\substack{i=1 \\ p \nmid i}}^{p^{t}}\left(X^{2^{m} p^{j}}+\delta^{i}\right), & \text { 若 } n>t .\end{cases}
$$

下面的引理给出 $X^{2^{m}}+\vartheta^{j}$ 在 $F_{q}$ 上的不可约分解, $0 \leqslant j \leqslant p^{n}-1$. 因此, 当 $n \leqslant t$ 时, 能得到 $X^{2^{m} p^{n}}+1$ 在 $F_{q}$ 上的不可约分解. 实际上, 用同样的方法也可以得到 $X^{2^{m}}+\delta^{k}$ 在 $F_{q}$ 上的不可约分 解, $0 \leqslant k \leqslant p^{t}-1$.

引理 3.6 假设 $n \leqslant t, \vartheta=\xi^{2 p^{t-n} c}$ 是 $F_{q}$ 中的一个本原 $p^{n}$ 次单位根, 则对每一个 $0 \leqslant j \leqslant p^{n}-1$, 都存在唯一的元素 $\varsigma_{j} \in\left\langle\xi^{2 c}\right\rangle$, 使得 $\varsigma_{j}^{2^{m}} \vartheta^{j}=1$. 特别地, 若令 $\varsigma_{p^{n}}=1$, 则 $\varsigma_{k}=\varsigma_{p^{n}-k}^{-1}, 0 \leqslant k \leqslant \frac{p^{n}-1}{2}$. 进 一步, 我们可以得到 $X^{2^{m}}+\vartheta^{j}$ 在 $F_{q}$ 上如下的不可约分解:

$$
X^{2^{m}}+\vartheta^{j}= \begin{cases}\vartheta^{j} \prod_{h \in H_{a}}\left(\left(\varsigma_{j} X\right)^{2^{m-a+1}}-2 h\left(\varsigma_{j} X\right)^{2^{m-a}}-1\right), & \text { 若 } m \geqslant a, \\ \vartheta^{j} \prod_{h \in H_{m}}\left(\left(\varsigma_{j} X\right)^{2}-2 h \varsigma_{j} X+1\right), & \text { 若 } m \leqslant a-1,\end{cases}
$$


其中 $h$ 和 $H_{m}$ 如引理 2.1 中所示.

证明 首先断言 $\vartheta^{j} \in\left\langle\xi^{2^{m}}\right\rangle, 0 \leqslant j \leqslant p^{n}-1$. 因为

$$
\operatorname{ord}\left(\xi^{2^{m}}\right)=\frac{q-1}{\operatorname{gcd}\left(q-1,2^{m}\right)}=\frac{2 p^{t} c}{\operatorname{gcd}\left(2 p^{t} c, 2^{m}\right)}=p^{t} c,
$$

所以, 循环子群 $\left\langle\xi^{2^{m}}\right\rangle$ 包含 $\langle\xi\rangle$ 的 Sylow $p$ - 子群. 因此, $\vartheta \in\left\langle\xi^{2^{m}}\right\rangle$, 即得 $\vartheta^{j} \in\left\langle\xi^{2^{m}}\right\rangle, 0 \leqslant j \leqslant p^{n}-1$, 断 言得证. 这样就存在某个整数 $z_{j}$, 使得 $\vartheta^{j}=\xi^{2^{m} z_{j}}$. 令 $\varsigma_{j}=\xi^{-z_{j}}$, 则有 $\varsigma_{j}^{2^{m}} \vartheta^{j}=\xi^{-2^{m} z_{j}} \vartheta^{j}=1$. 易知, $\varsigma_{j}$ 是 $\left\langle\xi^{2 c}\right\rangle$ 中满足 $\varsigma_{j}^{2^{m}} \vartheta^{j}=1$ 的唯一元素. 对每一个 $0 \leqslant j \leqslant p^{n}-1$, 我们能建立如下的 $F_{q^{-}}$代数同构:

$$
\begin{gathered}
\varphi_{j}: F_{q}[X] /\left\langle X^{2^{m}}+1\right\rangle \rightarrow F_{q}[X] /\left\langle X^{2^{m}}+\vartheta^{j}\right\rangle, \\
f(X)+\left\langle X^{2^{m}}+1\right\rangle \mapsto f\left(\varsigma_{j} X\right)+\left\langle X^{2^{m}}+\vartheta^{j}\right\rangle .
\end{gathered}
$$

根据引理 $2.1, X^{2^{m}}+1$ 在 $F_{q}$ 上有如下的不可约分解:

$$
X^{2^{m}}+1= \begin{cases}\prod_{h \in H_{a}}\left(X^{2^{m-a+1}}-2 h X^{2^{m-a}}-1\right), & \text { 若 } m \geqslant a, \\ \prod_{h \in H_{m}}\left(X^{2}-2 h X+1\right), & \text { 若 } m \leqslant(a-1) .\end{cases}
$$

通过同构 $\varphi_{j}$, 我们得到 $X^{2^{m}}+\vartheta^{j}$ 在 $F_{q}$ 上如下的不可约分解:

$$
X^{2^{m}}+\vartheta^{j}= \begin{cases}\vartheta^{j} \prod_{h \in H_{a}}\left(\left(\varsigma_{j} X\right)^{2^{m-a+1}}-2 h\left(\varsigma_{j} X\right)^{2^{m-a}}-1\right), & \text { 若 } m \geqslant a, \\ \vartheta^{j} \prod_{h \in H_{m}}\left(\left(\varsigma_{j} X\right)^{2}-2 h \varsigma_{j} X+1\right), & \text { 若 } m \leqslant(a-1) .\end{cases}
$$

引理 3.6 证毕.

根据引理 3.6, 我们容易得到下面的引理.

引理 3.7 符号如上. 设 $n \leqslant t, \vartheta=\xi^{2 p^{t-n} c}$ 是 $F_{q}$ 中的一个 $p^{n}$ 次单位根, 则 $X^{2^{m} p^{n}}+1$ 在 $F_{q}$ 上 的不可约分解如下:

$$
X^{2^{m} p^{n}}+1= \begin{cases}\prod_{j=0}^{p^{n}-1} \prod_{h \in H_{m}}\left(X^{2}-2 h \varsigma_{j}^{-1} X+\varsigma_{j}^{-2}\right), & \text { 若 } m \leqslant(a-1), \\ \prod_{j=0}^{p^{n}-1} \prod_{h \in H_{a}}\left(X^{2^{m-a+1}}-2 h \varsigma_{j}^{-2^{m-a}} X^{2^{m-a}}-\varsigma_{j}^{-2^{m-a+1}}\right), & \text { 若 } m \geqslant a,\end{cases}
$$

其中 $\varsigma_{j}$ 如引理 3.6 中所示, $h$ 和 $H_{m}$ 如注 2.1 中所示.

证明 当 $n \leqslant t$, 有

$$
X^{p^{n}}+1=(X+1)(X+\vartheta) \cdots\left(X+\vartheta^{p^{n}-1}\right) .
$$

因此,

$$
X^{2^{m} p^{n}}+1=\left(X^{2^{m}}+1\right)\left(X^{2^{m}}+\vartheta\right) \cdots\left(X^{2^{m}}+\vartheta^{p^{n}-1}\right) .
$$

根据引理 3.6, 我们即可得到所期望的结果.

当 $n>t$, 我们需要用下面的结果来刻画 $X^{2^{m} p^{n}}+1$ 在 $F_{q}$ 上的不可约分解. 


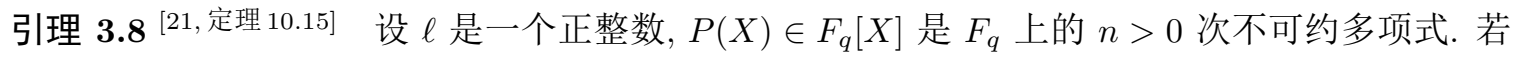
$P(0) \neq 0$, 且 $P(X)$ 的周期 $e$ 等于 $P(X)$ 的任一个根的阶数, 则 $P\left(X^{\ell}\right)$ 在 $F_{q}$ 上不可约当且仅当下面 的三个条件成立:

(1) $\ell$ 的每一个素因子均整除 $e$;

(2) $\operatorname{gcd}\left(\ell, \frac{q^{n}-1}{e}\right)=1$;

(3) 若 $4 \mid \ell$, 则 $4 \mid\left(q^{n}-1\right)$.

引理 3.9 符号如上. 设 $\delta=\xi^{2 c}$ 是一个本原 $p^{t}$ 次单位根.

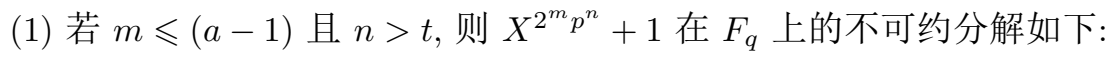

$$
X^{2^{m} p^{n}}+1=\prod_{k=0}^{p^{t}-1} \prod_{h \in H_{m}}\left(X^{2}-2 h \mu_{k}^{-1} X+\mu_{k}^{-2}\right) \cdot \prod_{j=1}^{n-t} \prod_{\substack{p^{t} \\ p \nmid i}}^{p_{h \in H_{m}}}\left(X^{2 p^{j}}-2 h \mu_{i}^{-1} X^{p^{j}}+\mu_{i}^{-2}\right) ;
$$

(2) 若 $m \geqslant a$ 且 $n>t$, 则 $X^{2^{m} p^{n}}+1$ 在 $F_{q}$ 上的不可约分解如下:

$$
\begin{aligned}
& X^{2^{m} p^{n}}+1=\prod_{k=0}^{p^{t}-1} \prod_{h \in H_{a}}\left(X^{2^{m-a+1}}-2 h \mu_{k}^{-2^{m-a}} X^{2^{m-a}}-\mu_{k}^{-2^{m-a+1}}\right) \\
& \times \prod_{j=1}^{n-t} \prod_{\substack{i=1 \\
p \nmid i}}^{p^{t}} \prod_{h \in H_{a}}\left(X^{2^{m-a+1} p^{j}}-2 h \mu_{i}^{-2^{m-a}} X^{2^{m-a} p^{j}}-\mu_{i}^{-2^{m-a+1}}\right),
\end{aligned}
$$

其中 $h$ 和 $H_{m}$ 如注 2.1 中所示, $\mu_{k}$ 是 $\langle\delta\rangle$ 中满足 $\mu_{k}^{2^{m}} \delta^{k}=1$ 的唯一元素, $0 \leqslant k \leqslant p^{t}-1$.

证明 (1) 根据引理 3.5 , 当 $n>t$ 时, 有如下分解:

$$
X^{p^{n}}+1=\prod_{k=0}^{p^{t}-1}\left(X+\delta^{k}\right) \cdot \prod_{j=1}^{n-t} \prod_{\substack{i=1 \\ p \nmid i}}^{p^{t}}\left(X^{p^{j}}+\delta^{i}\right)
$$

这里 $\delta=\xi^{2 c}$ 是一个本原 $p^{t}$ 次单位根. 因此,

$$
X^{2^{m} p^{n}}+1=\prod_{k=0}^{p^{t}-1}\left(X^{2^{m}}+\delta^{k}\right) \cdot \prod_{j=1}^{n-t} \prod_{\substack{i=1 \\ p \nmid i}}^{p^{t}}\left(X^{2^{m} p^{j}}+\delta^{i}\right) .
$$

利用引理 3.6 中所用的方法, 我们能得到多项式 $\prod_{k=0}^{p^{t}-1}\left(X^{2^{m}}+\delta^{k}\right)$ 在 $F_{q}$ 上的不可约分解. 下面断言 对任意的 $1 \leqslant j \leqslant n-t$ 和 $1 \leqslant i \leqslant p^{t}, p \nmid i$,

$$
X^{2^{m} p^{j}}+\delta^{i}=\delta^{i} \prod_{h \in H_{m}}\left(\mu_{i}^{2} X^{2 p^{j}}-2 h \mu_{i} X^{p^{j}}+1\right)
$$

是 $X^{2^{m} p^{j}}+\delta^{i}$ 在 $F_{q}$ 上的不可约分解. 既然

$$
X^{2^{m}}+1=\prod_{h \in H_{m}}\left(X^{2}-2 h X+1\right), \quad X^{2^{m}}+\delta^{i}=\delta^{i} \prod_{h \in H_{m}}\left(\mu_{i}^{2} X^{2}-2 h \mu_{i} X+1\right),
$$

因此, (3.2) 成立. 现在我们只需验证 (3.2) 右边的多项式因式在 $F_{q}$ 上不可约即可. 对于这一点, 我们 用引理 3.8 证明. 注意, 这里 $\ell=p^{j}, P(X)=\mu_{i}^{2} X^{2}-2 h \mu_{i} X+1$. 所以, 只需验证引理 3.8(1) 和 3.8(2) 成立即可. 令 $\beta$ 是 $P(X)$ 的任一根, 即 $\beta^{2^{m}}=-\delta^{i}$. 既然 $\delta$ 是一个本原 $p^{t}$ 次单位根, 且 $\operatorname{gcd}(p, i)=1$, 
易知, $2 p^{t} \mid \operatorname{ord}(\beta)$, 即 $2 p^{t} \mid e$. 显然, $\operatorname{gcd}(q+1, p)=1$. 所以, $p^{t} \|\left(q^{2}-1\right)$. 因此, 引理 3.8(1) 和 3.8(2) 成 立. 这就完成了引理 3.9(1) 的证明.

同理可证引理 3.9(2).

下面的定理刻画了有限域 $F_{q}$ 上所有长为 $2^{m} p^{n}$ 的负循环码, 这里 $p$ 是 $q-1$ 的一个奇素因子, 且 $4 \nmid(q-1)$. 为方便记, 我们总假设下面的 $\varepsilon_{i, k}^{j}$ 等于 0 或 $1, i, j$ 和 $k$ 分别取遍它们的下标和上标.

定理 3.10 符号如上. 设 $\vartheta=\xi^{2 p^{t-n} c}$ 是 $F_{q}$ 中一个本原 $p^{n}$ 次单位根 $(n \leqslant t), \delta=\xi^{2 c}$ 是一个本 原 $p^{t}$ 次单位根. 若 $4 \nmid(q-1)$, 则 $F_{q}$ 上所有长为 $2^{m} p^{n}$ 的负循环码为

(1) 若 $m \leqslant(a-1)$ 且 $n \leqslant t$, 则

$$
\left\langle\prod_{j=0}^{p^{n}-1} \prod_{h \in H_{m}}\left(X^{2}-2 h \varsigma_{j}^{-1} X+\varsigma_{j}^{-2}\right)^{\varepsilon_{j}^{h}}\right\rangle ;
$$

(2) 若 $m \geqslant a$ 且 $n \leqslant t$, 则

$$
\left\langle\prod_{j=0}^{p^{n}-1} \prod_{h \in H_{a}}\left(X^{2^{m-a+1}}-2 h \varsigma_{j}^{-2^{m-a}} X^{2^{m-a}}-\varsigma_{j}^{-2^{m-a+1}}\right)^{\varepsilon_{j}^{h}}\right\rangle
$$

(3) 若 $m \leqslant(a-1)$ 且 $n>t$, 则

$$
\left\langle\prod_{k=0}^{p^{t}-1} \prod_{h \in H_{m}}\left(X^{2}-2 h \mu_{k}^{-1} X+\mu_{k}^{-2}\right)^{\varepsilon_{k}^{h}} \prod_{j=1}^{n-t} \prod_{\substack{i=1 \\ p \nmid i}}^{p^{t}} \prod_{h \in H_{m}}\left(X^{2 p^{j}}-2 h \mu_{i}^{-1} X^{p^{j}}+\mu_{i}^{-2}\right)^{\varepsilon_{j, i}^{h}}\right\rangle ;
$$

(4) 若 $m \geqslant a$ 且 $n>t$, 则

$$
\begin{aligned}
& \left\langle\prod_{k=0}^{p^{t}-1} \prod_{h \in H_{a}}\left(X^{2^{m-a+1}}-2 h \mu_{k}^{-2^{m-a}} X^{2^{m-a}}-\mu_{k}^{-2^{m-a+1}}\right)^{\varepsilon_{k}^{h}}\right. \\
& \left.\quad \times \prod_{j=1}^{n-t} \prod_{\substack{i=1 \\
p \nmid i}}^{p^{t}} \prod_{h \in H_{a}}\left(X^{2^{m-a+1} p^{j}}-2 h \mu_{i}^{-2^{m-a}} X^{2^{m-a} p^{j}}-\mu_{i}^{-2^{m-a+1}}\right)^{\varepsilon_{j, i}^{h}}\right\rangle,
\end{aligned}
$$

这里 $\varsigma_{j}$ 是 $\langle\delta\rangle$ 中满足 $\varsigma_{j}^{2^{m}} \vartheta^{j}=1$ 的唯一元素, $0 \leqslant j \leqslant p^{n}-1, \mu_{k}$ 是 $\langle\delta\rangle$ 中满足 $\mu_{k}^{2^{m}} \delta^{k}=1$ 的唯一元素, $0 \leqslant k \leqslant p^{t}-1$.

\section{4 有限域 $F_{q}$ 上长为 $2^{m} p^{n}$ 的自对偶负循环码}

设 $F_{q}$ 是一个奇数阶有限域, 且它的乘法群 $F_{q}^{*}=\langle\xi\rangle$. 回忆 $q-1=2^{s} p^{t} c$, 这里 $s$ 和 $t$ 都是正整数, $p$ 是一个奇素数, $\operatorname{gcd}(2 p, c)=1$. 若 $s=1$, 则令 $2^{a} \|(q+1)$.

已经证明了奇特征有限域上的自对偶循环码是不存在的 [22]. 但是, 对于负循环码这个结论是不成 立的. 本节将得到 $F_{q}$ 上所有长为 $2^{m} p^{n}$ 的自对偶的负循环码的生成多项式以及它们的个数.

根据第 2 节对自对偶负循环码的讨论, 我们有下面的结果.

定理 4.1 符号如定理 3.3 中所示.

(1) 若 $m<s$ 且 $n \leqslant t$, 则 $F_{q}$ 上长为 $2^{m} p^{n}$ 的自对偶的负循环码恰好有 $2^{2^{m-1}} p^{n}$ 个, 它们是

$$
\left\langle\prod_{\substack{i=1 \\ 2 \nmid i}}^{2^{m} p^{n}}\left(X-\sigma^{i}\right)^{\varepsilon_{i}}\left(X-\sigma^{2^{m+1}} p^{n}-i\right)^{1-\varepsilon_{i}}\right\rangle
$$


(2) 若 $m \geqslant s$ 且 $n \leqslant t$, 则 $F_{q}$ 上长为 $2^{m} p^{n}$ 的自对偶的负循环码恰好有 $2^{2^{s-2} p^{n}}$ 个, 它们是

$$
\left\langle\prod_{\substack{i=1 \\ 2 \neq i}}^{2^{s-1} p^{n}}\left(X^{2^{m-s+1}}-\eta^{i}\right)^{\varepsilon_{i}}\left(X^{2^{m-s+1}}-\eta^{2^{s} p^{n}-i}\right)^{1-\varepsilon_{i}}\right\rangle
$$

(3) 若 $m<s$ 且 $n>t$, 则 $F_{q}$ 上长为 $2^{m} p^{n}$ 的自对偶的负循环码恰好有 $2^{\ell_{1}}$ 个, 这里

$$
\ell_{1}=2^{m-1} p^{t}+2^{m-1} p^{t-1}(n-t)(p-1)
$$

它们是

$$
\left\langle\prod_{\substack{k=1 \\ 2 \nmid k}}^{2^{m} p^{t}}\left(X-\sigma_{1}^{k}\right)^{\varepsilon_{k}}\left(X-\sigma_{1}^{2^{m+1}} p^{t}-k\right)^{1-\varepsilon_{k}} \prod_{i=0}^{n-t-1} \prod_{\substack{j=1 \\ p \nmid j, 2 \nmid j}}^{2^{m} p^{t}}\left(X^{p^{n-t-i}}-\sigma_{1}^{j}\right)^{\varepsilon^{j}}\left(X^{p^{n-t-i}}-\sigma_{1}^{2^{m+1} p^{t}-j}\right)^{1-\varepsilon_{i}^{j}}\right\rangle
$$

(4) 若 $m \geqslant s$ 且 $n>t$, 则 $F_{q}$ 上长为 $2^{m} p^{n}$ 的自对偶的负循环码恰好有 $2^{\ell_{2}}$ 个, 这里

$$
\ell_{2}=2^{s-2} p^{t}+2^{s-2} p^{t-1}(n-t)(p-1),
$$

它们是

$$
\begin{aligned}
\left\langle\prod_{\substack{u=1 \\
2 \nmid u}}^{2^{s-1} p^{t}}\left(X^{2^{m+1-s}}-\theta^{u}\right)^{\varepsilon_{u}}\left(X^{2^{m+1-s}}-\theta^{2^{s} p^{t}-u}\right)^{1-\varepsilon_{u}}\right. \\
\left.\quad \times \prod_{j=1}^{n-t} \prod_{\substack{r=1 \\
p \nmid r, 2 \nmid r}}^{2^{s-1} p^{t}}\left(X^{2^{m+1-s} p^{j}}-\theta^{r}\right)^{\varepsilon_{j}^{r}}\left(X^{2^{m+1-s} p^{j}}-\theta^{2^{s} p^{t}-r}\right)^{1-\varepsilon_{j}^{r}}\right\rangle .
\end{aligned}
$$

证明 根据引理 3.1, 我们知道, 若 $m<s$ 且 $n \leqslant t$, 则

$$
X^{2^{m} p^{n}}+1=\prod_{\substack{i=1 \\ 2 \nmid i}}^{2^{m+1} p^{n}}\left(X-\sigma^{i}\right)=\prod_{\substack{i=1 \\ 2 \nmid i}}^{2^{m} p^{n}}\left(X-\sigma^{i}\right)\left(X-\sigma^{2^{m+1} p^{n}-i}\right) .
$$

既然长为 $2^{m} p^{n}$ 的负循环码 $C$ 的生成多项式 $g(X)$ 是首一的并且整除 $X^{2^{m}} p^{n}+1$, 我们可以假设

$$
g(X)=\prod_{\substack{i=1 \\ 2 \nmid i}}^{2^{m} p^{n}}\left(X-\sigma^{i}\right)^{\epsilon_{i}}\left(X-\sigma^{2^{m+1} p^{n}-i}\right)^{\tau_{i}}
$$

这里 $\epsilon_{i}$ 等于 0 或 1 . 因此, $C$ 的校验多项式是

$$
h(X)=\prod_{\substack{i=1 \\ 2 \nmid i}}^{2^{m} p^{n}}\left(X-\sigma^{i}\right)^{1-\epsilon_{i}}\left(X-\sigma^{2^{m+1} p^{n}-i}\right)^{1-\tau_{i}},
$$

这里 $\epsilon_{i}, \tau_{i}$ 等于 0 或 1 . 进而即得

$$
h(X)^{*}=\prod_{\substack{i=1 \\ 2 \nmid i}}^{2^{m} p^{n}}\left(X-\sigma^{i}\right)^{1-\tau_{i}}\left(X-\sigma^{2^{m} p^{n}-i}\right)^{1-\epsilon_{i}} .
$$


注意到 $C^{\perp}=\left\langle h(X)^{*}\right\rangle$, 因此, $\tau_{i}+\epsilon_{i}=1$, 对任意的 $1 \leqslant i \leqslant 2^{m} p^{n}$ 而 $2 \nmid i$. 由此可以得到第一个结 论, 其余的结论同理可证.

为方便计, 我们采用如下的符号. 在注 2.1 中,

$$
H_{a}=\left\{ \pm\left(\frac{h-1}{2}\right)^{\frac{q+1}{4}} \mid h \in H_{a-1}\right\}
$$

由此我们令

$$
H_{a}^{+}=\left\{\left(\frac{h-1}{2}\right)^{\frac{q+1}{4}} \mid h \in H_{a-1}\right\}
$$

因此, $\left|H_{a}^{+}\right|=2^{a-2}$.

定理 4.2 符号如定理 3.10 中所示.

(1) 若 $m \leqslant(a-1)$, 则 $F_{q}$ 上长为 $2^{m} p^{n}$ 的自对偶的负循环码不存在;

(2) 若 $m \geqslant a$ 且 $n \leqslant t$, 则 $F_{q}$ 上长为 $2^{m} p^{n}$ 的自对偶的负循环码恰好有 $2^{\ell_{3}}$, 这里

$$
\ell_{3}=2^{a-2}+\frac{p^{n}-1}{2} \cdot 2^{a-1}
$$

它们是

$$
\left\langle\prod_{h \in H_{a}^{+}}\left(X^{2^{m-a+1}}-2 h X^{2^{m-a}}-1\right)^{\epsilon_{h}}\left(X^{2^{m-a+1}}+2 h X^{2^{m-a}}-1\right)^{1-\epsilon_{h}} \prod_{j=1}^{\frac{p^{n}-1}{2}} \prod_{h \in H_{a}} P_{j}^{h}(X)\right\rangle,
$$

这里

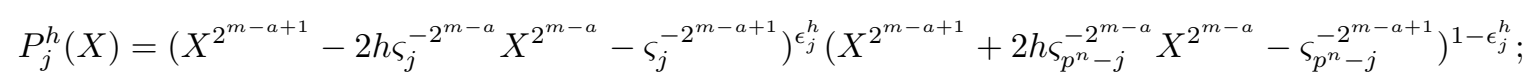

(3) 若 $m \geqslant a$ 且 $n>t$, 则 $F_{q}$ 上长为 $2^{m} p^{n}$ 的自对偶的负循环码恰好有 $2^{\ell_{4}}$, 这里

$$
\ell_{4}=2^{a-2}+\frac{p^{t}-1}{2} \cdot 2^{a-1}+2^{a-2}(n-t)\left(p^{t}-p^{t-1}\right),
$$

它们是

$$
\begin{gathered}
\left\langle\prod_{h \in H_{a}^{+}}\left(\left(X^{2^{m-a+1}}-2 h X^{2^{m-a}}-1\right)^{\epsilon_{h}}\left(X^{2^{m-a+1}}+2 h X^{2^{m-a}}-1\right)^{1-\epsilon_{h}}\right)\right. \\
\left.\quad \times \prod_{k=1}^{\frac{p^{t}-1}{2}} \prod_{h \in H_{a}} R_{k}^{h}(X) \cdot \prod_{\substack{i=1 \\
p \nmid i}}^{\frac{p^{t}-1}{2}} \prod_{j=1}^{n-t} \prod_{h \in H_{a}} Q_{j, i}^{h}(X)\right\rangle,
\end{gathered}
$$

这里

$$
\begin{aligned}
R_{k}^{h}(X)= & \left(X^{2^{m-a+1}}-2 h \mu_{k}^{-2^{m-a}} X^{2^{m-a}}-\mu_{k}^{-2^{m-a+1}}\right)^{\epsilon_{k}^{h}}\left(X^{2^{m-a+1}}+2 h \mu_{p^{t}-k}^{-2^{m-a}} X^{2^{m-a}}-\mu_{p^{t}-k}^{-2^{m-a+1}}\right)^{1-\epsilon_{k}^{h}}, \\
Q_{j, i}^{h}(X)= & \left(X^{2^{m-a+1} p^{j}}-2 h \mu_{i}^{-2^{m-a}} X^{2^{m-a} p^{j}}-\mu_{i}^{-2^{m-a+1}}\right)^{\varepsilon_{j, i}^{h}} \\
& \times\left(X^{2^{m-a+1} p^{j}}+2 h \mu_{p^{t}-i}^{-2^{m-a}} X^{2^{m-a} p^{j}}-\mu_{p^{t}-i}^{-2^{m-a+1}}\right)^{1-\varepsilon_{j, i}^{h}} .
\end{aligned}
$$

证明 我们仅给出 (1) 的证明. 若 $m \leqslant(a-1)$, 则根据注 $2.1, X^{2^{m}}+1$ 在 $F_{q}$ 上的每一个不可约 因式都是自反的. 显然, $X^{2^{m}}+1$ 是 $X^{2^{m} p^{n}}+1$ 的一个因式. 因此, $X^{2^{m} p^{n}+1}$ 有自反的不可约因式. 根 据第 2 节对自对偶负循环码的讨论, 得证. 
注 4.3 设 $F_{q}$ 是 $q$ 元有限域, 这里 $q \equiv 3(\bmod 4), n^{\prime}$ 是一个与 $q$ 互素的奇数. 如对定理 4.2(1) 的处理, 我们容易得到当 $e \leqslant(a-1)$ 时, $F_{q}$ 上长为 $2^{e} n^{\prime}$ 的自对偶的负循环码不存在, 这里 $a$ 是 2 整 除 $q+1$ 的最高次幂. 这个结果推广了文献 [17, 定理 3$]$.

\section{5 例子}

本节给出两个例子来说明文中的主要结果.

例 5.1 构造有限域 $F_{13}$ 上所有长为 $2^{m} 3^{n}$ 的负循环码, 这里 $q=13, p=3, s=2, t=1$ 和 $c=1$. 设 $\xi$ 是一个本原 12 次单位根. 根据定理 $3.3, F_{13}$ 上所有长为 $2^{m} 3^{n}$ 的负循环码为

(1) 若 $m=1$ 且 $n=1$, 则

$$
\left\langle\prod_{\substack{i=1 \\ 2 \nmid i}}^{12}\left(X-\xi^{i}\right)^{\varepsilon_{i}}\right\rangle
$$

(2) 若 $m \geqslant 2$ 且 $n=1$, 则

$$
\left\langle\prod_{\substack{j=1 \\ 2 \nmid j}}^{12}\left(X^{2^{m-1}}-\xi^{j}\right)^{\varepsilon_{j}}\right\rangle
$$

(3) 若 $m=1$ 且 $n>1$, 则

$$
\left\langle\prod_{\substack{k=1 \\ 2 \nmid k}}^{12}\left(X-\xi^{k}\right)^{\varepsilon_{k}} \cdot \prod_{i=0}^{n-2} \prod_{\substack{j=1 \\ 3 \nmid j, 2 \nmid j}}^{12}\left(X^{3^{n-1-i}}-\xi^{j}\right)^{\varepsilon_{i}^{j}}\right\rangle ;
$$

(4) 若 $m \geqslant 2$ 且 $n>1$, 则

$$
\left\langle\prod_{\substack{u=1 \\ 2 \nmid u}}^{12}\left(X^{2^{m-1}}-\theta^{u}\right)^{\varepsilon_{u}} \cdot \prod_{j=1}^{n-1} \prod_{\substack{r=1 \\ 2 \nmid r, 3 \nmid r}}^{12}\left(X^{2^{m-1} p^{j}}-\theta^{r}\right)^{\varepsilon_{j}^{r}}\right\rangle .
$$

再依据定理 $4.1, F_{13}$ 上所有长为 $2^{m} 3^{n}$ 的自对偶负循环码为

(1) 若 $m=1$ 且 $n=1$, 则 $F_{13}$ 上长为 6 的自对偶负循环码恰好有 8 个, 它们是

$$
\left\langle\prod_{\substack{i=1 \\ 2 \nmid i}}^{6}\left(X-\xi^{i}\right)^{\varepsilon_{i}}\left(X-\xi^{12-i}\right)^{1-\varepsilon_{i}}\right\rangle
$$

(2) 若 $m \geqslant 2$ 且 $n=1$, 则 $F_{13}$ 上长为 $2^{m} \cdot 3$ 的自对偶负循环码恰好有 8 个, 它们是

$$
\left\langle\prod_{\substack{i=1 \\ 2 \nmid i}}^{6}\left(X^{2^{m-1}}-\xi^{i}\right)^{\varepsilon_{i}}\left(X^{2^{m-1}}-\xi^{12-i}\right)^{1-\varepsilon_{i}}\right\rangle ;
$$

(3) 若 $m=1$ 且 $n>1$, 则 $F_{13}$ 上长为 $2 \cdot 3^{n}$ 的自对偶负循环码恰好有 $2^{\ell_{1}}$ 个, 这里 $\ell_{1}=3+2(n-1)$, 它们是

$$
\left\langle\prod_{\substack{k=1 \\ 2 \nmid k}}^{6}\left(X-\xi^{k}\right)^{\varepsilon_{k}}\left(X-\xi^{12-k}\right)^{1-\varepsilon_{k}} \prod_{i=0}^{n-2} \prod_{\substack{j=1 \\ 3 \nmid j, 2 \nmid j}}^{6}\left(X^{3^{n-1-i}}-\xi^{j}\right)^{\varepsilon_{i}^{j}}\left(X^{3^{n-1-i}}-\xi^{12-j}\right)^{1-\varepsilon_{i}^{j}}\right\rangle
$$


(4) 若 $m \geqslant 2$ 且 $n>1$, 则 $F_{13}$ 上长为 $2^{m} p^{n}$ 的自对偶负循环码恰好有 $2^{\ell_{2}}$ 个, 这里 $\ell_{2}=3+2(n-1)$, 它们是

$$
\left\langle\prod_{\substack{u=1 \\ 2 \nmid u}}^{6}\left(X^{2^{m-1}}-\xi^{u}\right)^{\varepsilon_{u}}\left(X^{2^{m-1}}-\xi^{12-u}\right)^{1-\varepsilon_{u}} \prod_{j=1}^{n-1} \prod_{\substack{r=1 \\ 3 \nmid r, 2 \nmid r}}^{6}\left(X^{2^{m-1} 3^{j}}-\xi^{r}\right)^{\varepsilon_{j}^{r}}\left(X^{2^{m-1} 3^{j}}-\xi^{12-r}\right)^{1-\varepsilon_{j}^{r}}\right\rangle .
$$

例 5.2 构造有限域 $F_{11}$ 上所有长为 $2^{m} 5^{n}$ 的负循环码, 这里 $q=11, p=5, a=2, t=1$ 和 $c=1$. 设 $\xi$ 是 $F_{11}$ 中一个本原 10 次单位根, 取 $\delta=\xi^{2}$ 是 $F_{11}$ 中一个本原 5 次单位根. 根据注 $2.1, H_{1}=\{0\}$, $H_{2}=\left\{\left(\frac{1}{2}\right)^{\frac{q+1}{4}},-\left(\frac{1}{2}\right)^{\frac{q+1}{4}}\right\}, H_{2}^{+}=\left\{\left(\frac{1}{2}\right)^{\frac{q+1}{4}}\right\}$. 根据定理 3.10 , 有限域 $F_{11}$ 上所有长为 $2^{m} 5^{n}$ 的负循环码为

(1) 若 $m=1$ 且 $n=1$, 则

$$
\left\langle\prod_{j=0}^{4}\left(X^{2}+\varsigma_{j}^{-2}\right)^{\varepsilon_{j}}\right\rangle
$$

(2) 若 $m \geqslant 2$ 且 $n=1$, 则

$$
\left\langle\prod_{j=0}^{4} \prod_{h \in H_{2}}\left(X^{2^{m-1}}-2 h \varsigma_{j}^{-2^{m-2}} X^{2^{m-2}}-\varsigma_{j}^{-2^{m-1}}\right)^{\varepsilon_{j}^{h}}\right\rangle
$$

(3) 若 $m=1$ 且 $n>1$, 则

$$
\left\langle\prod_{k=0}^{4}\left(X^{2}+\varsigma_{k}^{-2}\right)^{\varepsilon_{k}} \prod_{j=1}^{n-1} \prod_{i=1}^{4}\left(X^{2 p^{j}}+\varsigma_{i}^{-2}\right)^{\varepsilon_{i}^{j}}\right\rangle
$$

(4) 若 $m \geqslant 2$ 且 $n>1$, 则

$$
\begin{aligned}
\left\langle\prod_{k=0}^{4}\right. & \prod_{h \in H_{2}}\left(X^{2^{m-1}}-2 h \varsigma_{k}^{-2^{m-2}} X^{2^{m-2}}-\varsigma_{k}^{-2^{m-1}}\right)^{\varepsilon_{k}^{h}} \\
& \left.\times \prod_{j=1}^{n-t} \prod_{i=1}^{4} \prod_{h \in H_{2}}\left(X^{2^{m-1} 5^{j}}-2 h \varsigma_{i}^{-2^{m-2}} X^{2^{m-2} 5^{j}}-\varsigma_{i}^{-2^{m-1}}\right)^{\varepsilon_{j, i}^{h}}\right\rangle,
\end{aligned}
$$

这里 $\varsigma_{j}$ 是 $F_{11}^{*}$ 的 Sylow 5 - 子群中满足 $\varsigma_{j}^{2^{m}} \delta^{j}=1$ 的唯一元素, $0 \leqslant j \leqslant 4$.

再根据定理 4.1, 有限域 $F_{11}$ 上所有长为 $2^{m} 5^{n}$ 的自对偶负循环码为

(1) 若 $m=1$, 则 $F_{11}$ 上长为 $2 \cdot 5^{n}$ 的负循环码不存在;

(2) 若 $m \geqslant 2$ 且 $n=1$, 则 $F_{11}$ 上长为 $2^{m} \cdot 5$ 的自对偶负循环码恰好有 $2^{5}$ 个, 它们是

$$
\left\langle\prod_{h \in H_{2}^{+}}\left(X^{2^{m-1}}-2 h X^{2^{m-2}}-1\right)^{\epsilon_{h}}\left(X^{2^{m-1}}+2 h X^{2^{m-2}}-1\right)^{1-\epsilon_{h}} \prod_{j=1}^{2} \prod_{h \in H_{2}} P_{j}^{h}(X)\right\rangle,
$$

这里 $P_{j}^{h}(X)=\left(X^{2^{m-1}}-2 h \varsigma_{j}^{-2^{m-2}} X^{2^{m-2}}-\varsigma_{j}^{-2^{m-1}}\right)^{\epsilon_{j}^{h}}\left(X^{2^{m-1}}+2 h \varsigma_{5-j}^{-2^{m-2}} X^{2^{m-2}}-\varsigma_{5-j}^{-2^{m-1}}\right)^{1-\epsilon_{j}^{h}}$;

(3) 若 $m \geqslant 2$ 且 $n>1$, 则 $F_{11}$ 上长为 $2^{m} p^{n}$ 的自对偶负循环码恰好有 $2^{5+4(n-1)}$ 个, 它们是

$$
\left\langle\prod_{h \in H_{2}^{+}}\left(\left(X^{2^{m-1}}-2 h X^{2^{m-2}}-1\right)^{\epsilon_{h}}\left(X^{2^{m-1}}+2 h X^{2^{m-2}}-1\right)^{1-\epsilon_{h}}\right) \prod_{k=1}^{2} \prod_{h \in H_{2}} R_{k}^{h}(X) \cdot \prod_{i=1}^{2} \prod_{j=1}^{n-1} \prod_{h \in H_{2}} Q_{j, i}^{h}(X)\right\rangle,
$$

这里

$$
R_{k}^{h}(X)=\left(X^{2^{m-1}}-2 h \varsigma_{k}^{-2^{m-2}} X^{2^{m-2}}-\varsigma_{k}^{-2^{m-1}}\right)^{\epsilon_{k}^{h}}\left(X^{2^{m-1}}+2 h \varsigma_{5^{n}-k}^{-2^{m-2}} X^{2^{m-2}}-\varsigma_{5^{n}-k}^{-2^{m-1}}\right)^{1-\epsilon_{k}^{h}},
$$




$$
Q_{j, i}^{h}(X)=\left(X^{2^{m-1} 5^{j}}-2 h \mu_{i}^{-2^{m-2}} X^{2^{m-2} 5^{j}}-\mu_{i}^{-2^{m-1}}\right)^{\varepsilon_{j, i}^{h}}\left(X^{2^{m-1} 5^{j}}+2 h \mu_{5-i}^{-2^{m-2}} X^{2^{m-2} 5^{j}}-\mu_{5-i}^{-2^{m-1}}\right)^{1-\varepsilon_{j, i}^{h}}
$$

致谢 感谢评审专家的宝贵意见和建议使本文的质量有了进一步的提高.

\section{参考文献}

1 Berlekamp E R. Negacyclic codes for the Lee metric. In: Proceedings of the Conference on Combinatorial Mathematics and its Applications. Chapel Hill, NC: University of North Carolina Press, 1968, 298-316

2 Berlekamp E R. Algebraic Coding Theory (Revised edition). Laguna Hills: Aegean Park Press, 1984

3 Blackford T. Negacyclic codes over $Z_{4}$ of even length. IEEE Trans Inform Theory, 2003, 6: 1417-1424

4 Blackford T. Negacyclic duadic codes. Finite Fields Appl, 2008, 14: 930-943

5 Chen B, Fan Y, Lin L, et al. Constacyclic codes over finite fields. Finite Fields Appl, 2012, 18: 1217-1231

6 Dinh H Q, Lopez-Permouth S R. Cyclic and negacyclic codes over finite chain rings. IEEE Trans Inform Theory, 2004, 8: $1728-1744$

7 Dinh H Q. Complete distances of all negacyclic codes of length $2^{s}$ over $Z_{2^{a}}$. IEEE Trans Inform Theory, 2007, 1: $147-161$

8 Dinh H Q. On the linear ordering of some classes of negacyclic and cyclic codes and their distance distributions. Finite Fields Appl, 2008, 14: 22-40

9 Dinh H Q. Repeated-root constacyclic codes of length $2 p^{s}$. Finite Fields Appl, 2012, 18: 133-143

10 Kai X, Zhu S. Negacyclic self-dual codes over finite chain rings. Des Codes Cryptogr, 2012, 62: 161-174

11 Wolfmann J. Negacyclic and cyclic codes over $Z_{4}$. IEEE Trans Inform Theory, 1999, 7: 2527-2532

12 Zhu S, Kai X. Dual and self-dual negacyclic codes of even length over $Z_{2^{a}}$. Discrete Math, 2009, 309: 2382-2391

13 Zhu S, Qian K, Kai X. A note on negacyclic self-dual codes over $Z_{2^{a}}$. Discrete Math, 2012, 312: 3270-3275

14 Zhu S X, Kai X S. Negacyclic codes over Galois rings of characteristic $2^{a}$. Sci China Math, 2012, 55: 869-879

15 Huffman W C, Pless V. Fundamentals of Error Correcting Codes. Cambridge: Cambridge University Press, 2003

16 Bakshi G K, Raka M. A class of constacyclic codes over a finite field. Finite Fields Appl, 2012, 18: 362-377

17 Bakshi G K, Raka M. Self-dual and self-orthogonal negacyclic codes of length $2 p^{n}$ over a finite field. Finite Fields Appl, 2013, 19: 39-54

18 Benger N, Scott M. Constructing tower extensions of finite fields for implementation of pairing-based cryptography. In: Lecture Notes in Computer Science, vol. 6087. Berlin: Springer, 2010, 180-195

19 Blake I F, Gao S, Mullin R C. Explicit factorization of $X^{2^{k}}+1$ over $F_{p}$ with prime $p \equiv 3(\bmod 4)$. Appl Algebra Engrg Comm Comput, 1993, 4: 89-94

20 Lidl R, Niederreiter H. Finite Fields. Cambridge: Cambridge University Press, 2008

21 Wan Z. Lectures on Finite Fields and Galois Rings. Singapore: World Scientific Publishing, 2003

22 Jia Y, Ling S, Xing C. On self-dual cyclic codes over finite fields. IEEE Trans Inform Theory, 2011, 57: 2243-2251

\section{Negacyclic codes of length $2^{m} p^{n}$ over a finite field}

\section{SHEN RuLin, ZHANG GuangHui \& CHEN BoCong}

Abstract Let $F_{q}$ be a finite field of odd order $q$. The polynomial generators of all negacyclic codes of length $2^{m} p^{n}$ over $F_{q}$ are obtained, where $p$ is an odd prime divisor of $q-1$ and $m, n$ are positive integers; all self-dual negacyclic codes of length $2^{m} p^{n}$ over $F_{q}$ are also precisely characterized.

Keywords negacyclic code, irreducible factorization, polynomial generator, dual code

MSC(2010) 11T71, 94B15

doi: $10.1360 / 012013-148$ 